\title{
环境激励下的旋转机械工作模态参数辩识 ${ }^{*}$
}

\author{
李启行 褚福䂞 \\ (清华大学机械工程系 北京 100084)
}

\begin{abstract}
摘要 : 伴随着高速透平机械向大跨度、柔性结构方向发展, 转子-轴承系统的稳定性将面临着严峻考验。在出厂测试阶段, 确 保机组转子系统具有足够稳定性裕度是降低生产现场机组发生失稳故障风险的重要手段。采用适用于随机平稳环境激励下的 随机子空间法, 对机组的模态参数进行辨识, 可规避在转子非驱动端增设电磁激振器的传统测试方法。通过分析转子振型进 动方向，区分一阶正反进动的模态参数。结合 3- $\sigma$ 统计聚类算法, 剔除非稳定的噪声或物理极点, 形成了区分转子系统的正 反进动的稳态图。数值仿真表明, 随机子空间法可以有效地辨识系统的模态参数, 利用旋转机械的振型进动方向分析方法可 以区分正反进动。此外, 通过传统扫频激励模态参数辨识试验，验证了随机子空间方法的辨识精度和工程测试可行性。研究 结果可为透平机组的稳定性测试提供技术和理论支撑。
\end{abstract}

关键词：透平机械；工作模态辨识；随机激励；转子稳定性

中图分类号 : TK47

\section{Operational Modal Identification for Rotating Machinery under Environment Excitation}

\author{
LI Qihang CHU Fulei
}

(Department of Mechanical Engineering, Tsinghua University, Beijing 100084)

\begin{abstract}
With the development of high-speed turbo machinery with the large span and flexible structure, the rotordynamic stability of the rotor-bearing system faces server challenges. The shop test to ensure the unit with an enough rotordynamic stability margin is an important process to reduce the risk of the instability fault of field units. Using the stochastic subspace identification (SSI) method, which is suitable for the random stationary environment excitations, to identify the modal parameters of units can avoid the traditional method that needs an additional electromagnetic exciter installed at the none-drive end of the rotor. The first forward and backward modal parameters are distinguished by analyzing the whirl directions of modal shapes. The unstable noise and physical poles are eliminated by combining the $3-\sigma$ clustering algorithm, and the stabilization diagram that can distinguish the forward and backward whirl modes of rotor system is also obtained. The numerical simulation results indicate that the SSI method can effectively identify the system modal parameters, and forward and backward whirl modes can be distinguished with the whirl direction analysis method of modal shapes in rotating machinery. Moreover, the traditional modal parameters identification method with sine-swept excitations is used to experimentally validate the identification accuracy of SSI method and its feasibility for industrial tests. The study result can provide technical and theoretical support for the stability test for turbo machinery units.
\end{abstract}

Key words : turbo machinery ; opeartional modal identification ; random excitation ; rotordynamic stability

\section{0 前言}

随着流程工业的生产装置的机组容量不断扩大， 高速透平机械(如离心压缩机、汽轮机、燃机发电机 组等)将面临着高参数化(高转速、高功率、高流量、 高压比)，机械结构向大跨度、柔性结构方向发展。 这就使得机组变得更加挠性 , 机组极易发生失稳故

* 国家自然科学基金(11802153，51335006)和中国博士后科学基金 (2018M631451)资助项目。20181018 收到初稿, 20190430 收到修改稿
障, 转子-轴承系统的稳定性将面临着严峻考验 ${ }^{[1]}$ 。

国内外均发生了因机组未进行稳定性裕度测试 评价工作而出现的机组失稳事故 ${ }^{[2-3]}$, 所以对转子系 统的稳定性评估测试工作十分必要。目前, 针对透 平机组的稳定性参数测试主要以转子非驱动端附加 电磁给力器进行激励测试系统的振动衰减信号或频 响函数的方式来辨识系统的稳定性参数 ${ }^{[4-5]}$, 即一阶 正进动模态的阻尼比(对数衰减率)。但是转子系统 有别于一般机械结构, 因轴系旋转而产生的陀螺效 应及支撑的各向异性会导致系统成对出现固有频率 
接近的正反进动模态。通过复数域信号描述方式构 造直接频响函数 (Directional frequency response function, $\mathrm{dFRF})^{[6]}$, 并在正、负频率轴上进行模态辨 识可实现正反进动的分离。李启行等 ${ }^{[7]}$ 和 TAKAHASHI 等 ${ }^{[8]}$ 利用电磁轴承扫频激励进行了试验测试研究, 验证了复数域下正、反进动分离的特性。此外, 采 用电磁激励器给转子施加固定频率的正/反向激励 力, 测量激振停止后转子的振动衰减信号, 并采用 反向自回归方法也可实现对一阶正/反进动的模态 参数辨识 ${ }^{[9]}$ 。但是以上方法的弊端是需要在设计阶 段考虑附加激振器的轴段, 而国内已有的成型机组 在设计时并未考虑。并且附加的激励装置会改变转 子原始结构造成系统模态参数发生变化, 实际现场 也不具备改造条件，因此具有一定局限性。因此， 如何避开外加激励装置的方式, 利用转子工作状态 时自身环境激励的振动响应对转子系统进行稳定性 参数辨识具有现实的工程实际应用价值。

GUGLIELMO 等 ${ }^{[10]}$ 利用压缩机机组存在的环 境激励(如气流激励力), 采用随机子空间法识别振 动信号的相关函数对机组的工作模态进行辨识, 但 在稳态图上并不能实现自动区分正反进动，需要人 为根据振型轨迹进行判断。因此, 本文采用透平旋 转机械正反进动振型来区分固有频率十分接近的 正反进动模态。同时，针对传统功率谱矩阵的奇异 值分解(Singular value decomposition, SVD)曲线因 正反进动模态叠加而不能准确凸显正反进动固有 频率的问题, 文中借用直接频响函数( $\mathrm{dFRF}$ ) 分离正 反进动模态的思想, 提出了直接功率谱函数 (Directional power spectra density function, dPSDF) 概念, 实现了功率谱函数的正反进动成分分离, 为基于协方差驱动的随机子空间法的稳态图提供 了区分正反进动模态的依据。此外, 通过将构造 两种不同维度的矩阵来获得的极点进行对比, 剔 除相同阶次下的虚假模态及不稳定模态。利用正 反进动模态判据和三倍标准差 (3- $\sigma)$ 统计聚类方 法, 剔除异常极点从而实现正反进动的极点的自 动聚类, 获得统计平均值作为模态参数的估计值。 数值和试验研究验证了方法的精度和工程测试可 行性, 研究结果可为高端压缩机机组稳定性可靠 设计、透平机组稳定性测试及稳定性在线状态监 测提供理论支撑。

\section{1 模态参数辨识理论}

1.1 离散系统方程及协方差性质 仅有随机白噪声输入的离散系统状态方程为

$$
\left\{\begin{array}{l}
\boldsymbol{x}_{k+1}=\boldsymbol{A} \boldsymbol{x}_{k}+\boldsymbol{B} \boldsymbol{w}_{k} \\
\boldsymbol{y}_{k}=\boldsymbol{C} \boldsymbol{x}_{k}
\end{array}\right.
$$

式中 $\boldsymbol{x}_{k}$ 一状态变量, 系统阶次为 $p$;

$\boldsymbol{y}_{k}$ 一输出变量, 通道数为 $l$;

$\boldsymbol{w}_{k}$ 一均值为 $\boldsymbol{O}$ 的白噪声序列;

$\boldsymbol{A}$ - 状态转移矩阵 ;

$\boldsymbol{B}, \boldsymbol{C}$ 一输入、输出矩阵。

假定变量 $\boldsymbol{x}_{k} 、 \boldsymbol{y}_{k}$ 与白噪声序列 $\boldsymbol{w}_{k}$ 的期望满足

$$
\begin{gathered}
E\left(\boldsymbol{x}_{k} \boldsymbol{w}_{k}^{\mathrm{T}}\right)=\frac{1}{j} \sum_{k=0}^{j-1} \boldsymbol{x}_{k} \boldsymbol{w}_{k}^{\mathrm{T}}=\boldsymbol{0} \\
E\left(\boldsymbol{y}_{k} \boldsymbol{w}_{k}^{\mathrm{T}}\right)=\frac{1}{j} \sum_{k=0}^{j-1} \boldsymbol{C} \boldsymbol{x}_{k} \boldsymbol{w}_{k}^{\mathrm{T}}=\mathbf{0}
\end{gathered}
$$

根据式(1)迭代关系有

$$
\boldsymbol{x}_{k+i}=\boldsymbol{A}^{i} \boldsymbol{x}_{k}+\sum_{m=0}^{i-1} \boldsymbol{A}^{m} \boldsymbol{w}_{k}
$$

定义输出序列的协方差矩阵

$$
\boldsymbol{R}_{i}=E\left(\boldsymbol{y}_{k+i} \boldsymbol{y}_{k}^{\mathrm{T}}\right)=\lim _{j \rightarrow \infty} \frac{1}{j} \sum_{k=0}^{j-1} \boldsymbol{y}_{k+i} \boldsymbol{y}_{k}^{\mathrm{T}}
$$

针对有限测点情况，将式(1)、(3)代入式(4)有

$$
\begin{gathered}
\boldsymbol{R}_{i}=\frac{1}{j} \sum_{k=0}^{j-1} \boldsymbol{C} \boldsymbol{x}_{k+i} \boldsymbol{y}_{k}^{\mathrm{T}}= \\
\frac{1}{j} \sum_{k=0}^{j-1}\left(\boldsymbol{C} \boldsymbol{A}^{i} \boldsymbol{x}_{k} \boldsymbol{y}_{k}^{\mathrm{T}}+\sum_{m=0}^{i-1} \boldsymbol{C} \boldsymbol{A}^{m} \boldsymbol{B} \boldsymbol{w}_{k} \boldsymbol{y}_{k}^{\mathrm{T}}\right)= \\
\boldsymbol{C} \boldsymbol{A}^{i} \cdot \mathrm{E}\left(\boldsymbol{x}_{k} \boldsymbol{y}_{k}^{\mathrm{T}}\right)+\sum_{m=0}^{i-1} \boldsymbol{C} \boldsymbol{A}^{m} \boldsymbol{B} \cdot \mathrm{E}\left(\boldsymbol{w}_{k} \boldsymbol{y}_{k}^{\mathrm{T}}\right)
\end{gathered}
$$

结合式(2)，则式(5)可简化为

$$
\boldsymbol{R}_{i}=\boldsymbol{C} \boldsymbol{A}^{i} \cdot \mathrm{E}\left(\boldsymbol{x}_{k} \boldsymbol{y}_{k}^{\mathrm{T}}\right)
$$

1.2 协方差驱动的随机子空间法

定义具有 $j$ 列的由输出序列 $\boldsymbol{y}_{k}$ 组成的“过去” 矩阵 $\boldsymbol{Y}_{p}$ 如下 ${ }^{[11]}$

$$
\boldsymbol{Y}_{p}=\boldsymbol{Y}_{0 i-1}=\frac{1}{\sqrt{j}}\left[\begin{array}{llll}
\boldsymbol{y}_{0} & \boldsymbol{y}_{1} & \cdots & \boldsymbol{y}_{j-1} \\
\boldsymbol{y}_{1} & \boldsymbol{y}_{2} & \cdots & \boldsymbol{y}_{j} \\
\vdots & \vdots & & \vdots \\
\boldsymbol{y}_{i-1} & \boldsymbol{y}_{i} & \cdots & \boldsymbol{y}_{i+j-2}
\end{array}\right]_{i \mid \times j}
$$

式中，下标 $m \mid n$ 表示第 1 行第 1 个元素为 $\boldsymbol{y}_{m}$, 最后 1 行第 1 个元素为 $\boldsymbol{y}_{n}$ 。

则“未来” 矩阵可以描述为

$$
\boldsymbol{Y}_{f}=\boldsymbol{Y}_{i \mid 2 i-1}, \boldsymbol{Y}_{f+1}=\boldsymbol{Y}_{i+1 \mid 2 i}
$$

定义 Toeplitz 矩阵

$$
\boldsymbol{T}_{1 \mid i}=\boldsymbol{Y}_{f} \boldsymbol{Y}_{p}^{\mathrm{T}}, \boldsymbol{T}_{2 \mid i+1}=\boldsymbol{Y}_{f+1} \boldsymbol{Y}_{p}^{\mathrm{T}}
$$

结合式(4)中协方差矩阵的定义, 式(9)为 


$$
\boldsymbol{T}_{1 \mid i}=\left[\begin{array}{cccc}
\boldsymbol{R}_{i} & \boldsymbol{R}_{i-1} & \cdots & \boldsymbol{R}_{1} \\
\boldsymbol{R}_{i+1} & \boldsymbol{R}_{i} & \cdots & \boldsymbol{R}_{2} \\
\vdots & \vdots & & \vdots \\
\boldsymbol{R}_{2 i-1} & \boldsymbol{R}_{2 i-2} & \cdots & \boldsymbol{R}_{i}
\end{array}\right]
$$
写为

此外, 结合式(4)定义, Toeplitz 矩阵 $\boldsymbol{T}_{1 \mid i}$ 可以改

$$
T_{1 \mid i}=\Gamma \Theta
$$

式中, $\boldsymbol{\Gamma}=\left[\begin{array}{c}\boldsymbol{C} \\ \boldsymbol{C} \boldsymbol{A} \\ \vdots \\ \boldsymbol{C} \boldsymbol{A}^{i-1}\end{array}\right], \Theta=\left[\begin{array}{llll}\boldsymbol{A}^{i} & \boldsymbol{A}^{i-1} & \cdots & \boldsymbol{A}\end{array}\right] \mathrm{E}\left(\boldsymbol{x}_{k} \boldsymbol{y}_{k}^{\mathrm{T}}\right)$ 。

对 Toeplitz 矩阵 $\boldsymbol{T}_{1 \mid i}$ 进行 SVD 分解有

$$
\boldsymbol{T}_{1 \mid i}=\left[\begin{array}{ll}
\boldsymbol{U}_{1} & \boldsymbol{U}_{2}
\end{array}\right]\left[\begin{array}{ll}
\boldsymbol{S}_{1} & \\
& \boldsymbol{S}_{2}=\mathbf{0}
\end{array}\right]\left[\begin{array}{l}
\boldsymbol{V}_{1}^{\mathrm{T}} \\
\boldsymbol{V}_{2}^{\mathrm{T}}
\end{array}\right]=\boldsymbol{U}_{1} \boldsymbol{S}_{1} \boldsymbol{V}_{1}^{\mathrm{T}}
$$

式中, $\boldsymbol{S}_{1}$ 的维度即为系统阶次 $p$, 取估计值

$$
\hat{\Gamma}=U_{1} S_{1}^{1 / 2} \quad \hat{\Theta}=S_{1}^{1 / 2} V_{1}^{\mathrm{T}}
$$

另外，从式(9)、(11)可获得

$$
\boldsymbol{T}_{2 \mid i+1}=\Gamma A \Theta
$$

因此，通过式(13)、(14)可求得状态矩阵 $\boldsymbol{A}$

$$
\boldsymbol{A}=\left(\boldsymbol{S}_{1}^{-1 / 2} \boldsymbol{U}_{1}^{\mathrm{T}}\right) \boldsymbol{T}_{2 \mid i+1}\left(\boldsymbol{V}_{1} \boldsymbol{S}_{1}^{-1 / 2}\right)
$$

其中, $C$ 矩阵可由矩阵 $\Gamma$ 的前 $l$ 行获得，其中 $l$ 为输出的通道数。

\section{3 辨识系统参数及振型}

求解离散系统的状态转移矩阵 $\boldsymbol{A}$ 的特征根及特 征矢量

$$
\boldsymbol{A}=\boldsymbol{\phi} \Lambda \phi^{-1}
$$

式中， $\boldsymbol{\Lambda}=\operatorname{diag}\left\{z_{1}, z_{2}, \cdots, z_{s}\right\}, z_{i}$ 是离散方程的特征根， $\phi$ 为特征矢量矩阵。

系统的固有频率和阻尼比为

$$
\begin{aligned}
& f_{i}=\left|\lambda_{i}\right| / 2 \pi \\
& \xi_{i}=-\operatorname{Re}\left(\lambda_{i}\right) /\left|\lambda_{i}\right|
\end{aligned}
$$

式中， $\lambda_{i}=\ln \left(z_{i}\right) / \Delta t, \Delta t$ 为采样时间间隔。

其连续模型对应的振型为

$$
\Phi=C \phi
$$

\section{4 转子系统正反进动判断准则}

转子某截面的振动信号为

$$
\begin{aligned}
& x(t)=a \angle \varphi_{a}=a \cos \left(\omega t+\varphi_{a}\right) \\
& y(t)=b \angle \varphi_{b}=b \cos \left(\omega t+\varphi_{b}\right)
\end{aligned}
$$

式中 $x(t), y(t)-x, y$ 方向的振动信号；

$a, b$ 振动幅值 ; $\varphi_{a}, \varphi_{b} \longrightarrow$ 初始相位；

$\omega$ 一振动角频率。

利用欧拉公式

$$
\cos (\omega t)=\frac{1}{2}(\exp (-\mathrm{j} \omega t)+\exp (\mathrm{j} \omega t))
$$

则复数信号 $z=x+\mathrm{j} \boldsymbol{y}$ 可分解为

$$
\boldsymbol{z}=\boldsymbol{z}_{b} \exp (-\mathrm{j} \omega t)+\boldsymbol{z}_{f} \exp (\mathrm{j} \omega t)
$$

式中, $\boldsymbol{z}_{f}$ 和 $z_{b}$ 分别是正反进动的初始复数矢量, 其 具体表达式为

$$
\begin{aligned}
& \boldsymbol{z}_{b}=\frac{1}{2}\left(a \exp \left(-\mathrm{j} \varphi_{a}\right)+\mathrm{j} b \exp \left(-\mathrm{j} \varphi_{b}\right)\right) \\
& \boldsymbol{z}_{f}=\frac{1}{2}\left(a \exp \left(\mathrm{j} \varphi_{a}\right)+\mathrm{j} b \exp \left(\mathrm{j} \varphi_{b}\right)\right)
\end{aligned}
$$

若 $\left|\boldsymbol{z}_{f}\right|>\left|\boldsymbol{z}_{b}\right|$, 则转子为正进动; 反之, $\left|\boldsymbol{z}_{f}\right|<\left|z_{b}\right|$ 为反进动。通过化简有: $\sin \left(\varphi_{a}-\varphi_{b}\right)>0$ 时, 为正 进动; $\sin \left(\varphi_{a}-\varphi_{b}\right)<0$ 时, 为反进动。

利用该判据即可获得复模态振型的进动方向， 实现对转子正反进动模态的区分。

\section{5 复数域信号的功率谱 SVD 分解}

假定有 $n$ 个测振面，则测振面间的复数信号功 率谱矩阵为

$$
\boldsymbol{G}=\left[\begin{array}{cccc}
G_{11} & G_{12} & \ldots & G_{1 n} \\
G_{21} & G_{22} & \cdots & G_{2 n} \\
\vdots & \vdots & & \vdots \\
G_{n 1} & G_{n 2} & \cdots & G_{n n}
\end{array}\right]
$$

式(22)中，功率谱矩阵满足

$$
G_{i j}=\left\{\begin{array}{cc}
\operatorname{psd}\left(\boldsymbol{z}_{i}, \boldsymbol{z}_{j}\right), & i=j \\
\operatorname{cpsd}\left(\boldsymbol{z}_{i}, \boldsymbol{z}_{j}\right), & i \neq j
\end{array}\right.
$$

式中 psd一自功率谱函数 ;

cpsd一互功率谱函数 ;

$z$ 一测振平面的复数信号。

对其每个频率点进行 SVD 分解有

$$
\boldsymbol{G}(\omega)=\boldsymbol{U} \boldsymbol{S} \boldsymbol{V}^{\mathrm{T}}
$$

式中， $\boldsymbol{S}=\operatorname{diag}\left\{S_{1}(\omega), S_{2}(\omega), \cdots, S_{n}(\omega)\right\}$ 。

其中， $S_{n}(\omega)$ 依次对应按信号能量降序排序排列 的全谱自功率谱，且当 $\omega>0$ 时，对应的是正进动振 动成分， $\omega<0$ 时，对应的是反进动振动成分。

\section{2 数值仿真验证}

\section{1 随机激励仿真计算}

为了验证基于随机环境激励的转子一阶正进动 模态参数辨识方法的理论精度。对美国弗吉尼亚大 学 ROMAC 实验室的稳定性测试试验台(图 1a)，建 
立了图 $1 \mathrm{~b}$ 所示的转子有限元模型, 转子的质量为 $83.63 \mathrm{~kg}$, 模型参数参见文献[9]。该转子由两个五 瓦可倾瓦油膜轴承支撑(节点 10 和 59), 并且轴上有 三个电磁轴承(节点 4、22、47)可作为激振器。仿真 所采用的轴承参数如表 1 所示。通过建立有限元模 型进行模态分析, 可获得系统在转速为 $8000 \mathrm{r} / \mathrm{min}$ 时, 理论一阶反正进动模态参数分别为: $f_{1 B}=87.07$ $\mathrm{Hz}, \xi_{1 B}=9.43 \%$ 和 $f_{1 F}=88.13 \mathrm{~Hz}, \xi_{1 F}=1.78 \%$ 。

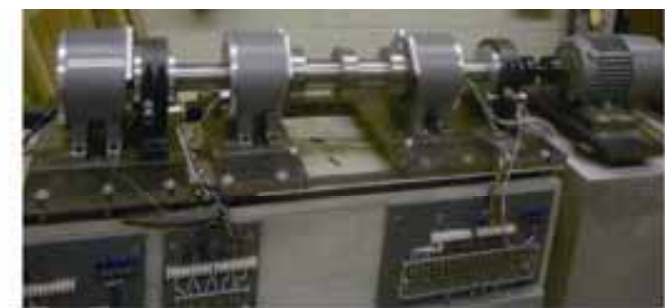

(a) 转子实物图

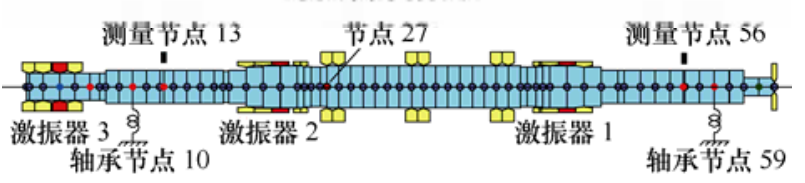

(b) 有限元模型及测点位置

图 1 试验转子系统的结构及有限元模型

表 1 仿真所用的轴承参数

\begin{tabular}{ccccc}
\hline \multirow{2}{*}{ 轴承 } & \multicolumn{5}{c}{ 刚度 $/\left(\mathrm{MN} \cdot \mathrm{m}^{-1}\right)$} \\
\cline { 2 - 5 } & $k_{x x}$ & $k_{x y}$ & $k_{y x}$ & $k_{y y}$ \\
\hline 节点 10 & 60.40 & -0.12 & 0.14 & 62.36 \\
节点 59 & 59.59 & -0.19 & 0.22 & 61.44 \\
\hline \hline 轴承 & \multicolumn{5}{c}{ 阻尼 $/\left(\mathrm{N} \cdot \mathrm{s} \cdot \mathrm{mm}^{-1}\right)$} \\
\cline { 2 - 5 } & $c_{x x}$ & $c_{x y}$ & $c_{y x}$ & $c_{y y}$ \\
\hline 节点 10 & 83.03 & 0.12 & -0.14 & 83.27 \\
节点 59 & 64.69 & 0.16 & -0.27 & 64.96 \\
\hline
\end{tabular}

节点 27 施加不平衡量为 $0.8 \mathrm{~g} \cdot \mathrm{mm}$ 模拟不平衡 振动, 对激振器 1 所在节点 47 施加最大幅值为 $20 \mathrm{~N}$ 的随机激励力模拟环境激励力, 工作转速为 8000 $\mathrm{r} / \mathrm{min}$, 仿真 $100 \mathrm{~s}$ 数据, 取后 $60 \mathrm{~s}$ 稳定振动的数据 进行分析(采样频率为 $2048 \mathrm{~Hz}$ )。用于模态分析的 信号为轴承测点节点 13 和 56 的 $x 、 y$ 方向振动信号, 节点 13 的全谱图如图 2 所示, 微弱的正、反进动响 应成分在约 $\pm 88 \mathrm{~Hz}$ 处激励起来了。

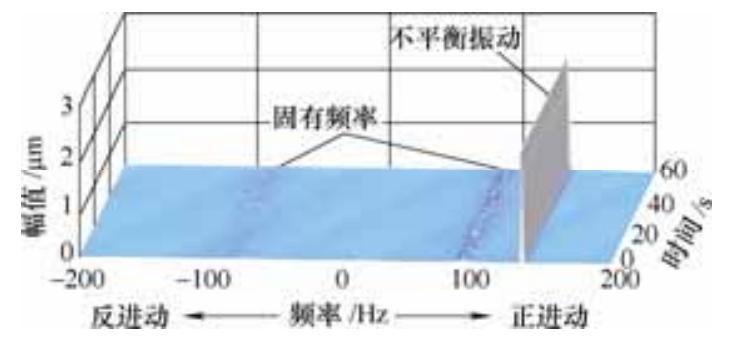

图 2 左轴承仿真的瀑布图

\section{2 仿真信号的模态参数辨识}

(1) 未处理的稳态图。为满足协方差驱动的随 机子空间算法的识别需求, 模型阶次 $i$ 满足 $i>p / l$, 且列数需满足 $j>20 i^{[11]}$ 。数值仿真中，振动数据取 两个轴承测点(节点 13 和 56)的 $x$ 和 $y$ 方向的数据， 则总通道数 $l=4$ 。若定最大分析阶次为 100 , 则 $\boldsymbol{Y}_{p}$ 矩阵的最小行数 $i_{\text {min }}=25$ 。假定每通道的信号总采样 点为 $N$, 则利用所有数据点来构造矩阵 $\boldsymbol{Y}_{p}, \boldsymbol{Y}_{p}$ 和 $\boldsymbol{Y}_{p+1}$ 的行数为 $j=N-2 i$ 。针对仿真数取后 $60 \mathrm{~s}$ 数据, 其长度 $N=2048 \mathrm{~Hz} \times 60 s=122880$, 取构造矩阵行数 $i=60$, 则 $j=122760$ 。利用协方差驱动的随机子空间 识别方法, 获得极点图及功率谱矩阵 SVD 分解最大 值的曲线如图 3 所示。可见图 3 中存在众多的虚假 模态，比较杂乱，给工程人员辨识转子系统的模态 造成困扰。

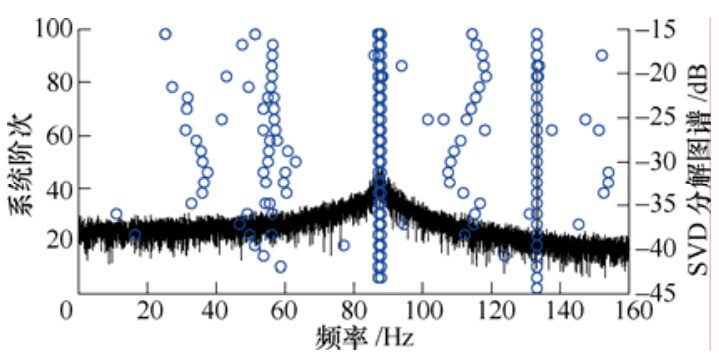

图 3 含传统功率谱矩阵分解曲线的稳态图

（2）极点对比剔除法。借鉴张永祥等 ${ }^{[11]}$ 提出的 模态自动识别算法的思路，构造两种不同矩阵 $\left(i_{A}=60\right.$ 和 $i_{B}=40$ ), 计算并将两次辨识的结果(分别对 应 $A 、 B$ 两组结果)进行对比, 剔除不稳定的极点, 主要依据就是稳定极点受构造矩阵行数影响较小, 而计算模态或虚假模态受其影响较大，其基本思路 如下。

判断系统阶次 $p$ 时, $A$ 组中第 $m$ 个极点对应的 模态参数组合点 $\left(f_{p, m}^{A}, \xi_{p, m}^{A}\right)$ 为稳定极点的判据就 是 : $B$ 组中至少存在一个点 $\left(f_{p, n}^{B}, \xi_{p, n}^{B}\right)$ 位于参考点 $\left(f_{p, m}^{A}, \xi_{p, m}^{A}\right)$ 为中心的判据椭圆内 , 即

$$
\begin{aligned}
& \left(\frac{\left|f_{m}^{A}-f_{n}^{B}\right|}{\Delta f}\right)^{2}+\left(\frac{\left|\xi_{m}^{A}-\xi_{n}^{B}\right|}{\Delta \xi_{0}}\right)^{2}<1 \\
& \Delta f=\min \left(\delta_{f} \max \left(f_{p, m}^{A}, f_{p, n}^{B}\right), \Delta f_{0}\right)
\end{aligned}
$$

式中 $f$ 一极点对应的固有频率 ;

$\xi$ 一极点对应的阻尼比 ;

$\delta_{f}$ 一频率相对偏差，取值 5\%;

$\Delta f_{0}$ 一频率绝对偏差，取值 $2 \mathrm{~Hz}$;

$\Delta \xi_{0}$ 一阻尼比绝对偏差, 取值 $0.5 \%$ 。

(3) 3- $\sigma$ 统计聚类方法。由于旋转机械的特殊性， 存在固有频率十分接近的正反进动模态, 且存在不 
平衡响应等谐响应激励。因此, 需要利用模态振型 对正反进动加以区分，避免频率接近造成误识别。 因此, 采用以下步骤, 分别展开针对正、反进动模 态的聚类统计辨识。

(1) 正进动模态分类。利用模态振型将极点分为 两类 : 正进动 $A_{f}$ 和反进动 $A_{b}$ 。

(2) 对正进动 $A_{f}$ 进行分类聚类。聚类规则: 若 $A_{f}$ 中任意 $i, j$ 两点中, 满足式(27)则归为一类, 最 终 $A_{f}$ 被分成 $k$ 类, $A_{f}=\left\{A_{f 1}, A_{f 2}, \cdots, A_{f k}\right\}$ 。不同类别间 的元素均不满足判据式(27)

$$
\left(\frac{\left|f_{i}^{A}-f_{j}^{A}\right|}{\Delta f_{0}}\right)^{2}+\left(\frac{\left|\xi_{i}^{A}-\xi_{j}^{A}\right|}{\Delta \xi_{0}}\right)^{2}<1
$$

(3) 剔除少元素的类别。设定极点个数 $P_{0}$, 集 合 $A_{f}=\left\{A_{f 1}, A_{f 2}, \cdots, A_{f k}\right\}$ 中，每一类的元素个数若小 于设定值 $P_{0}$ 则判定为不稳定极点, 予以剔除。假定 计算阶次 $p$ 的总数为 $n$, 考虑真实的阻尼模态极点 是一对共轭复数, 具有相同的频率和阻尼比, 所以 阈值的取值为 $P_{0}=[2 n / 3],[\cdot]$ 表示向上取整。

(4) 三倍标准差聚类篮选。对每一类辨识获得值 进行统计计算, 获得辨识获得的频率和阻尼比的统 计值, 然后利用式(28)的聚类法则，保留以均值为 中心的 3 倍标准差椭圆以内的点

$$
\frac{(f-\bar{f})^{2}}{\left(3 \sigma_{f}\right)^{2}}+\frac{(\xi-\bar{\xi})^{2}}{\left(3 \sigma_{\xi}\right)^{2}}<1
$$

式中， $\sigma$ 表示标准差，上标“一”表示均值。

通过反复迭代，可以剔除异常值，确保最终所 有的点满足式(28), 即全部落在 3 倍标准差椭圆之 内。统计均值则作为最终的模态参数估计值。

(5) 对反进动 $A_{b}$ 的极点重复步骤(2) (4), 即可 获得反进动模态中每一类的统计值。

(4) 统计聚类后的正反进动稳态图。通过聚类, 剔除虚假模态及不稳定模态, 并在 $160 \mathrm{~Hz}$ 内辨识获 得了图 4 中所示的 3 类极点。利用获得测量截面复 数信号的功率谱矩阵 SVD 分解曲线, 绘制如图 4 所示。在 $88 \mathrm{~Hz}$ 附近, 正进动曲线比反进动曲线陡

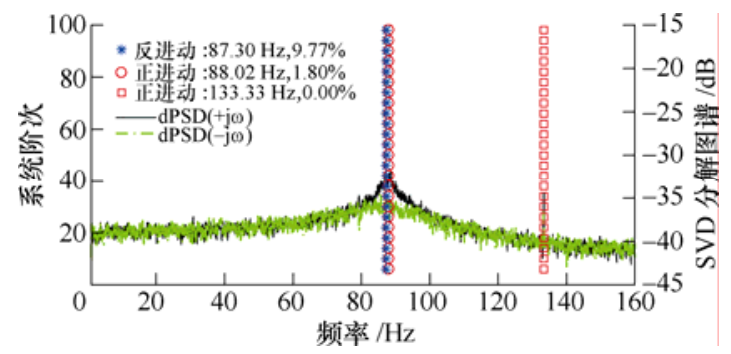

图 4 剔除虚假模态及区分正反进动的稳态图
峭一些, 说明正进动的阻尼比小于反进动的阻尼比。 $133 \mathrm{~Hz}$ 处的陡峭尖峰则是转子不平衡质量激励所 致。结合 SVD 分解曲线可以看出, 较为陡峭的正进 动功率谱分解曲线对应阻尼比较小的正进动极点, 而较为平坦的反进动功率谱分解曲线对应阻尼比较 大的反进动极点。

此外，图 5 展示了统计聚类篮选后的固有频率 和阻尼比散点分布图。在该坐标平面下，可以清楚 地看出 3 类极点的分布情况, 其中 $133 \mathrm{~Hz}$ 对应的不 平衡激励频率, 其阻尼比几乎为 0 , 结合 SVD 分解 曲线中 $133 \mathrm{~Hz}$ 的尖峰可综合判断, $133 \mathrm{~Hz}$ 不是固 有频率，而是外部激励所致的谐响应模态。最终的 模态统计值为，反进动： $f_{1 B}=87.30 \mathrm{~Hz}, \xi_{1 B}=9.77 \%$; 正进动 : $f_{1 F}=88.02 \mathrm{~Hz}, \xi_{1 F}=1.80 \%$ 。

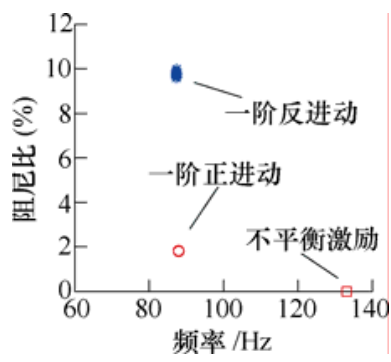

图 5 節选出来的极点的频率-阻尼比分布

通过数值仿真结果可以看出，环境激励下的协 方差随机子空间法可以区分正反进动模态参数，且 能稳定辨识出转子系统的一阶正反进动的模态参 数。同特征根分析的模态参数理论值对比可知, 利 用环境激励响应信号辨识获得的正进动阻尼比绝对 误差仅为 $0.02 \%$, 反进动的阻尼比绝对误差为 $0.34 \%$ 。反进动辨识结果相对正进动要差一些, 这 主要是因为在环境随机激励振动响应中，以正进动 为主; 而反进动振动成分所占比例较小, 其信号的 信噪比较低。尽管如此, 其相对误差均在 5\%以内, 可以满足工程测试需求。

\section{3 试验分析}

\section{1 试验过程介绍}

试验测试采用的是图 1a 所示的试验台，具体 的结构尺寸, 文献[9]进行了详尽的描述。本文试 验中, 转子工作在 $8000 \mathrm{r} / \mathrm{min}$, 轴承润滑油供油温 度 $42 \sim 43{ }^{\circ} \mathrm{C}$, 油压 $0.2 \mathrm{MPa}$ 。转子的降速时的伯 德图如图 6 所示。可以知道一阶临界转速约为 $4800 \mathrm{r} / \mathrm{min}$, 对应的频率约为 $80 \mathrm{~Hz}$ 。因此, 可以 判断在工作转速 $8000 \mathrm{r} / \mathrm{min}$ 时, 系统的固有频率 在 $80 \mathrm{~Hz}$ 附近。 


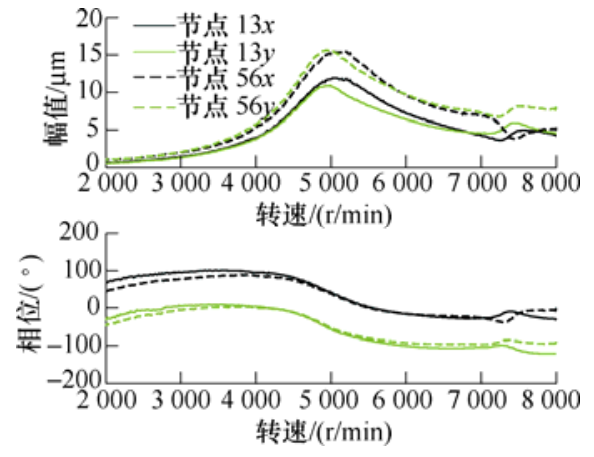

图 6 转子降速时的伯德图

\section{2 扫频试验结果}

图 $7 \mathrm{a}$ 和图 $7 \mathrm{~b}$ 分别是转子在 $8000 \mathrm{r} / \mathrm{min}$ 时利用 激振器 1 对转子进行正、反向激励的瀑布图, 激励 的频率从 $20 \mathrm{~Hz}$ 到 $200 \mathrm{~Hz}$ ，增加的步长为 $1 \mathrm{~Hz} / \mathrm{s}$ 。 利用转子稳定性参数识别方法 ${ }^{[12]}$, 处理扫频数据获 得的辨识结果为: 一阶正进动的模态参数, $f_{I F}=$ $78.27 \mathrm{~Hz}, \xi_{1 F}=5.28 \%$; 一阶反进动的模态参数， $f_{1 B}$ $=84.5 \mathrm{~Hz}, \xi_{1 B}=6.78 \%$ 。正因为一阶正进动的阻尼 比较小, 所以图 $7 \mathrm{~b}$ 中的正向激励响应的经过固有频 率时的幅值比图 7a 中的反向激励时的幅值陡峭。

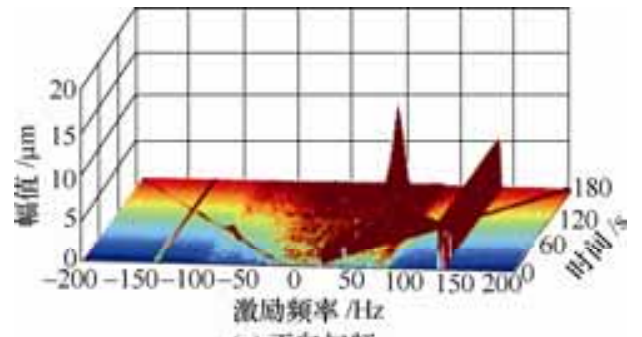

(a) 正向扫额

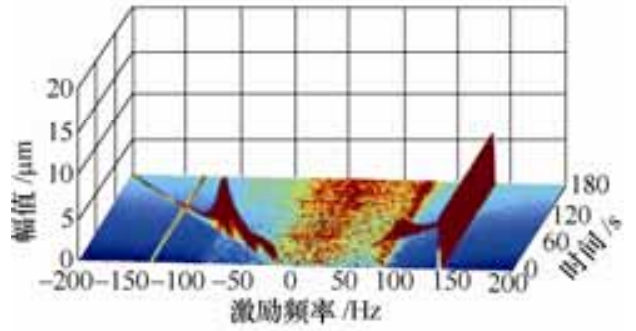

(b) 反向扫颣

图 7 扫频激励下的全谱瀑布图

\subsection{SSI 工作模态辨识结果}

在转速为 $8000 \mathrm{r} / \mathrm{min}$ 时, 测量节点 13 和 56 处 的振动信号作为系统输出。每通道的信号长度为 $60 \mathrm{~s}$, 信号采样率为 $3000 \mathrm{~Hz}$, 式(7)中的矩阵规模 为 $i=60, j=179880$ 。通过协方差驱动的随机子空间 辨识方法可以获得图 8 所示的稳态图。图 8 中的 SVD 分解图谱中, $133 \mathrm{~Hz}$ 对应的是转速频率。

采用第 2.2 节中的对比剔除及统计聚类篮选方 法获得图 9 所示的稳态图。在 $0 \sim 140 \mathrm{~Hz}$ 范围内, 仅有 3 类模态参数被识别出来, 其中 $133.25 \mathrm{~Hz}$ 对 应为转速激励频率，不是转子系统的固有频率。

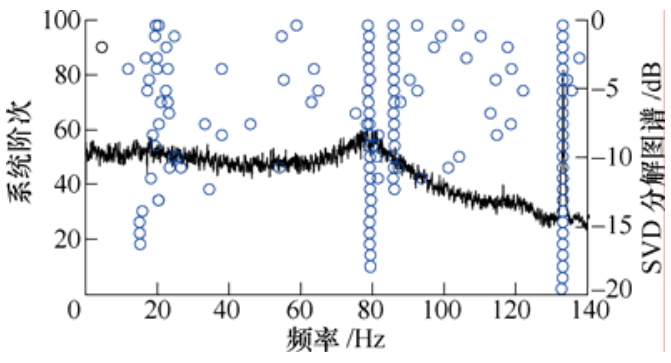

图 8 试验结果的原始稳态图

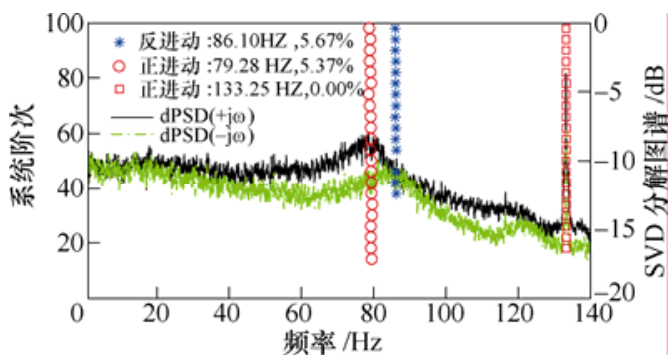

图 9 剔除虚假模态的实验结果的稳态

值得一提的是，采用传统的 SVD 分解曲线(图 8)，仅仅只能判断 $80 \mathrm{~Hz}$ 附近对应转子系统的一个 模态, 而不能判断邻近 $86 \mathrm{~Hz}$ 附近的模态来自哪里, 且不能判断模态的进动状态。相比之下, 在图 9 中 采用复数信号的 dPSDF 矩阵的 SVD 分解曲线, 可 以知道在频率区间 $70 \sim 90 \mathrm{~Hz}$ 内，dPSDF 的正负频 率曲线的峰值分别对应着正反进动的固有频率，易 于直观判断。此外，图 10 是统计聚类篮选后的固有 频率和阻尼比散点分布图，明显可以看到不同阶次 下的正反进动的频率和阻尼比分布情况。

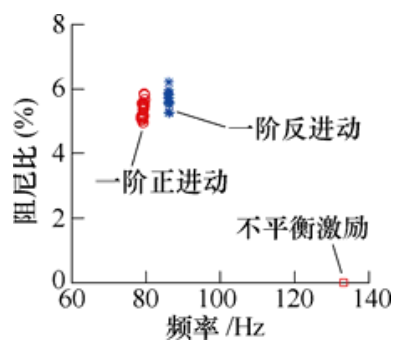

图 10 筛选出来的极点的频率-阻尼比分布

最终的模态参数统计均值为 $f_{1 F}=79.28 \mathrm{~Hz}, \xi_{1 F}=$ $5.37 \% ; f_{1 B}=86.10 \mathrm{~Hz}, \xi_{1 B}=5.67 \%$ 。同扫频激励辨 识的试验结果相比，利用随机激励响应信号获得的 一阶正进动的固有频率和阻尼比的绝对偏差均较 小, 分别为 $0.01 \mathrm{~Hz}$ 和 $0.09 \%$,一阶反进动模态参数 的绝对偏差稍微大一点, 分别为 $1.6 \mathrm{~Hz}$ 和 $1.11 \%$ 。

这表明利用随机激励辨识的一阶正进动模态参 数值得可信, 并且精度与外加激励器的测试结果相 比; 反进动的模态参数辨识结果相对较差一些。其 主要原因是，整个随机激励响应信号中的反进动模 态占比较小，信噪比较低。虽然如此，但是压缩机 主要测试其一阶正进动的阻尼比或对数衰减率来评 
价稳定性，反进动并不关心。

\section{4 结论}

(1) 结合旋转机械正、反进动模态振型的协方 差驱动的随机子空间法, 可以有效地区分固有频率 十分接近的一对正、反进动模态参数; 提出 $3-\sigma$ 聚 类篎选方法, 实现了物理模态参数的自动获取, 规 避人为选择引入的误差, 保障了识别结果的稳定性。

(2) 数值仿真算例中，工作模态辨识方法获得 模态参数与理论模型的模态分析结果一致, 理论验 证了工作模态辨识方法的精度, 一阶正进动的阻尼 比绝对误差仅为 $0.02 \%$ 。

(3) 通过传统的电磁激励扫频模态参数试验, 验证了随机子空间方法的试验辨识精度，与有源激 励辨识的结果相比, 其中辨识的一阶正进动阻尼比 绝对偏差仅为 $0.09 \%$ 。

(4) 本文提出的方法可为工程机组的出厂稳定 性测试、失稳故障在线监测预防及反馈机组设计提 供技术手段。

\section{参 考 文 献}

[1] 马辉, 李辉, 牛和强, 等. 滑动轴承-转子系统油膜失稳 参数影响分析 $[\mathrm{J}]$. 振动与冲击, $2013,32(23): 100-104$. MA Hui ,LI Hui ,NIU Heqiang, et al. Parametric influence analysis of oil-film instability in a sliding bearing-rotor system[J]. Journal of Vibration and Shock ,2013, 32(23) : 100-104.

[2] 梁国斌. 合成气压缩机改造后汽轮机振动原因分析 [J]. 大氮肥，2009，32(2):81-84.

LIANG Guobin. Reasons for steam turbine vibration after revamp of syngas compressor[J]. Large Scale Nitrogenous Fertilizer Industry , 2009 , 32(2) : 81-84.

[3] MOORE J J , MASSIMO P D , ANTHONY C , et al. Investigation of a rotordynamic instability in a high pressure centrifugal compressor due to damper seal clearance divergence[C]// 7th IFToMM-Conference on Rotor Dynamics ,September 25-28, 2006 ,Vienna ,Austria. $2006: 25-28$

[4] ZHANG D , LEE C , FALLER W, et al. Evaluation of bearing designs for a multistage centrifugal compressor using a magnetic exciter[C]// Proc. of the Fourty-first
Turbomachinery Symposium , September 24-27, 2012 , Houston ,Texas. Turbomachinery Laboratory ,Texas A\&M University , $2012: 1-10$.

[5] Pettinato B C , Cloud C H. CAMPOS R S. Shop acceptance testing of compressor rotordynamic stability and theoretical correlation[C]// Proc. of the Thirty-Ninth Turbomachinery Symposium , October 4-7 , 2010 , Houston ,Texas. Turbomachinery Laboratory ,Texas A\&M University , $2010: 31-42$.

[6] KESSLER C L. Complex modal analysis of rotating machinery[D]. Dayton : University of Dayton , 1991.

[7] 李启行, 王维民, 齐鹏逸，等. 转子轴承系统稳定性分 析与识别方法[J]. 机械工程学报 , $2014,50(7)$ : 54-59. LI Qihang, WANG Weimin, QI Pengyi, et al. Stability analysis and identification method for rotor-bearings system[J]. Journal of Mechanical Engineering, 2014 , 50(7) : 54-59.

[8] TAKAHASHI N, MAGARA Y, NARITA $\mathrm{M}$, et al. Rotordynamic evaluation of centrifugal compressor using electromagnetic exciter[J]. Journal of Engineering for Gas Turbines and Power , $2012,134(3)$ : 032505.

[9] CLOUD C H. Stability of rotors supported by tilting pad journal bearings[D]. Charlottesille : University of Virginia , 2007.

[10] GUGLIELMO A ,MITARITONNA N ,CATANZARO M , et al. Full load stability test (FLST) on LNG compressor [C]// ASME Turbo Expo 2014 : Turbine Technical Conference and Exposition ,June 16-20 ,2014. Düsseldorf , Germany. New York : ASME , 2014 : V07AT31A006.

[11］张永祥，刘心，褚志刚，等. 基于随机子空间法的模态 参数自动提取[J]. 机械工程学报, 2018,54(9): 187-194. ZHANG Yongxiang, LIU Xin, CHU Zhigang, et al. Autonomous modal parameter extraction based on stochastic subspace identification[J]. Journal of Mechanical Engineering , 2018 , 54(9) : 187-194.

[12] WANG W , LI Q , GAO J , et al. An identification method for damping ratio in rotor systems[J]. Mechanical Systems and Signal Processing , 2016 , 68 : 536-554.

作者简介: 李启行, 男, 1989 年出生, 博士后。主要研究方向为旋转机 械动力学与模态参数辨识。

E-mail : liqihang2012@foxmail.com

褚福否(通信作者), 男, 1959 年出生，教授，博士研究生导师。主要研 究方向为旋转机械动力学、机械故障诊断技术、非线性振动等。

E-mail : chufl@tsinghua.edu.cn 